\title{
A COMPARISON OF THE FORMALDEHYD-GEL REAC- TION OF GATE AND PAPACOSTAS WITH THE WASSERMANN REACTION *
}

\author{
VICTOR BURKE, PH.D.
}

SAN FRANCISCO

Gaté and Papacostas ${ }^{1}$ have recently described a new serum test for syphilis which in their hands gave results agreeing quite closely with the Wassermann reaction. The technic of the test as developed by them consists of adding two drops of solution of formaldehyd to 1 c.c. of clear serum in a test tube, shaking to obtain a thorough mixture, plugging the tubes with "cotton wool" stoppers, and allowing them to stand at room temperature for from twenty-four to thirty hours. Readings are then made; the serums which have become solid and jelly-like are considered as giving a positive reaction and those which remain fluid, a negative reaction. A comparison of this test with the Wassermann reaction on over 400 serums showed an agreement in 85 per cent. of cases. When the reactions differed the authors were unable to determine which was the more accurate. In early cases of syphilis in which the clinical diagnosis was positive and the Wassermann reaction negative, the formaldheyd-gel reaction was positive. The authors found that mixtures of positive serums always gave positive results and mixtures of negative serums negative results, that preliminary inactivation is unnecessary, and that the reaction is not modified by incubation.

It is evident that if this test for syphilis, either as described in the foregoing or in some modified form, can be depended on to give the results obtained by Gaté and Papacostas, the physician will have at his disposal a fairly satisfactory serum test for syphilis which can be made in his office as readily as in well equipped laboratories. The importance of a more thorough knowledge of this test is obvious.

In this paper I describe the results of a comparison of the formaldehyd-gel test with the Wassermann test as made in the Stanford Laboratory. The usual procedure followed in these experiments was to make the formaldehyd-gel test a day later than the Wassermann test. The Wassermann tests were the routine tests as made by the technicians four days a week in the Stanford Laboratory. The serums remaining were used on the following day for the formaldehyd-gel tests. Thus

* From the Stanford Laboratory, Stanford Medical School.

1. Gaté and Papacostas: Compet. rend. Soc. de Biol. 20:1432 (Nov.) 1920. 
the serums were always older when subjected to the formaldehyd-gel test, but none of them had been drawn more than five days, usually two or three days, and they were always kept on ice.

The routine Wassermann test as made in the Stanford Laboratory consists in the use of three antigens, an alcoholic extract of guinea-pig heart, a cholesterinized alcoholic extract of calf heart and an acetone insoluble extract of lamb heart. Primary incubation is done in the water bath for one hour. In special cases the icebox method is also used.

The formaldehyd-gel tests were made by adding solution of formaldehyd to the inactivated serums in the proportion of two drops of solution of formaldehyd to 1 c.c. of serum. There was some variation in the size of the drops. The tubes unstoppered were left in the room or placed in the incubator or in a closed chamber containing solution of formaldehyd. A few simple experiments indicated that the reaction was more rapid and in closer agreement with the Wassermann test when the tubes were left unstoppered and placed in the incubator, and this method was finally adopted. It is thus seen that the method used by us is a modification of the method described by Gate and Papacostas. We modified their method because in our hands it failed to detect a sufficiently large number of positive serums to serve as a practical test. This modification, which resulted in increased desiccation of the serum, may account for some of the false positives obtained by us.

Readings were made at twenty-four hour intervals. The reaction was considered positive when the serum became gelatinous and failed to flow when the tube was inverted and jarred against the hand. The readings were either positive or negative. Attempts to grade the reaction by estimating the degree of viscosity were not satisfactory as normal serums vary to a considerable degree in this respect. Occasionally the surface of the serum becomes membranous and adherent to the glass and a distinct jar is necessary to start the fluid flowing.

The effect of heat, desiccation and increased volatilization of the formaldehyd gas on the rate of coagulation and percentage of positive reactions is suggested by the following experiments:

\section{EXPERIMENTS WITH FORMALDEHYD}

Experiment 1.-One-half c.c. of a positive serum was placed in each of three serum tubes and one drop of solution of formaldehyd added to each. One tube was placed in the incubator, one in a closed chamber containing solution of formaldehyd, and the third exposed to the air on the desk. All the tubes were unstoppered. The tube in the incubator was coagulated on the fifth day. The other two tubes were not coagulated on the sixth day and were discarded. 
The experiment was repeated with a different positive serum. The tube in the incubator was coagulated in twenty-four hours, the tube in the formaldehyd vapor at forty-eight hours, and the tube exposed to the air of the room at seventy-two hours.

The experiment was repeated with still another positive serum, and all three tubes were coagulated in twenty-four hours.

Experiment 2.-One-half c.c. of a positive serum was placed in each of three serum tubes. One tube was closed with a cork stopper, one with a cotton stopper and the third tube was left unstoppered. All three tubes were placed in the incubator. The serum in the unstoppered tube and the cotton stoppered tube was coagulated on the fourth day. The serum in the cork stoppered tube was not coagulated on the sixth day and was discarded.

Experiment 3--One=half c.c. of a positive serum was placed in each of three serum tubes. One drop of solution of formaldehyd was added to the first tube, two drops to the second tube and six drops to the third tube. The tubes were placed in the incubator, unstoppered. The tubes containing one and two drops of solution of formaldehyd, respectively, were coagulated on the second day. The serum in the tube which received six drops was coagulated on the third day:

Expcriment 4.-One c.c. of a positive serum was placed in each of two serum tubes. Two drops of solution of formaldehyd were added to each tube. One tube was then placed in the incubator and the other in the icebox. Both tubes were unstoppered. The tube in the incubator was coagulated on the fifth day, while the tube in the icebox was not coagulated on the sixth day and was discarded.

Experiment 5.-One c.c. of a positive serum was placed in each of two test tubes ( 6 by $5 / 8$ inches [15.24 cm. by $15.85 \mathrm{~mm}$.]). Two drops of solution of formaldehyd were placed in one test tube. Both tubes were held over a low Bunsen burner flame. The serum without the solution of formaldehyd quickly coagulated. The serum to which the liquor formaldehydi had been added boiled vigorously for some time and did not coagulate. ${ }^{2}$

These experiments suggest that heat affects the formaldehyd coagulation of the serum only indirectly on account of increased evaporation. Increased evaporation is accompanied by increased volatilization of the formaldehyd gas. The effect of this is not known.

The number of positive serums obtained in the routine Wassermann tests and available to us averaged about 12.5 per cent., which gave a small percentage of positive serum to work with. This fact should be kept in mind in interpreting the results obtained.

\section{RESULTS OBTAINED WITH FORMALDEHYD TEST AND THOSE OBTAINED WITH WASSERMANN TEST}

A comparison of the results obtained with the formaldehyd test at different periods of time with the results of the Wassermann tests, using three different antigens, is given in Table 1 .

2. The possible application of this fact to the making of serum mediums for bacteriologic work will be determined later. 
An analysis of Table 1 shows that the results obtained with the formaldehyd-gel test, as made by us, agree with the results obtained with the Wassermann test in from 85 to 95 per cent. of the cases. The percentage varies with the time, number of serums used and the antigen, being greatest with the alcoholic extract antigen. The formaldehyd-gel test gave a negative reaction in from 91 to 98 per cent. of the serums giving a negative reaction with the Wassermann test. It gave positive reactions in from 12 to 70 per cent. of the serums, giving positive reactions with the Wassermann. The agreement with the positive Wassermann reaction was greatest when the formaldehydgel test was read at seventy-two hours and when an alcoholic extract antigen was used for the Wassermann test.

TABLE 1.-A Comparison of the Formaldehyd-Gel and Routine Wassermann Tests of Serums*

\begin{tabular}{|c|c|c|c|c|c|c|c|c|c|}
\hline \multirow{2}{*}{$\begin{array}{c}\text { Antigen } \\
\text { Duration of formaldehyd-gel test in } \\
\text { hours } \ldots \ldots \ldots \ldots \ldots \ldots \ldots \ldots \ldots \ldots \ldots\end{array}$} & \multicolumn{3}{|c|}{ Cholesterinized } & \multicolumn{3}{|c|}{ Acetone Insoluble } & \multicolumn{3}{|c|}{ Alcoholic Extraet } \\
\hline & 9 & 18 & & $?$ & 8 & 7 & & 48 & 79 \\
\hline 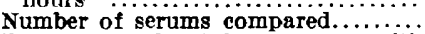 & 319 & 477 & 284 & 319 & $4 \frac{40}{47}$ & 284 & $\begin{array}{r}24 \\
319\end{array}$ & 478 & 284 \\
\hline Percentage of total agreement with & 86 & 87 & 85 & 04 & $\infty$ & 99 & 05 & O4 & 04 \\
\hline $\begin{array}{l}\text { Wassermann reaction } \ldots \ldots \ldots \ldots \\
\text { Percentage of agreement with } \text { Was- } \\
\text { sermann negativest } \ldots \ldots \ldots \ldots \ldots \ldots\end{array}$ & 98 & 98 & 96 & 97 & 91 & 96 & 97 & 96 & 96 \\
\hline $\begin{array}{l}\text { Percentage of agreement with Was- } \\
\text { sermann positives } \$ \ldots \ldots \ldots \ldots \ldots \ldots\end{array}$ & 12 & 16 & 21 & 16 & 21 & 40 & 22 & 25 & 70 \\
\hline
\end{tabular}

* Fractions ignored.

† Percentage of the number of negative or positive Wassermann reactions.

The agreement with the negative Wassermann reaction tends to be less at seventy-two hours than at twenty-four, owing to an increase in the number of false positives obtained with the formaldehyd. The agreement with the positive Wassermann reactions is greater at seventy-two than at twenty-four hours. In other words, the formaldehyd-gel test gives a larger percentage of true positive reactions and a larger percentage of false positive reactions ${ }^{3}$ at seventy-two than at twenty-four hours, the total agreement remaining about the same.

It is seen in Table 1 that the results obtained by the formaldehydgel test are in closer agreement with the results obtained by the Wassermann test with the alcoholic extract antigen than with the cholesterinized antigen. In Table 2 the results obtained with the alcoholic extract antigen and formaldehyd-gel test and those obtained with the cholesterinized antigen are compared. The results obtained with the alcoholic extract antigen are in closer agreement with the results

3. In speaking of true and false positive and negative reactions, we refer to the results obtained with the Wassermann test using a cholesterinized antigen as the standard, unless otherwise specified. The cholesterinized antigen gives a higher percentage of positive reactions, and the results are in closer agreement with the clinical histories of the patients than the results obtained with the other two antigens. 
obtained with the cholesterinized antigen than are the results obtained with the formaldehyd. In addition, the alcoholic extract antigen does not produce any false positives when compared with the cholesterinized antigen. Using the results obtained with the cholesterinized antigen as the standard, we must conclude that the formaldehyd-gel reaction is less reliable as a test for syphilis than the Wassermann test in which alcoholic extract of guinea-pig heart is used as antigen. And since the results obtained with the alcoholic extract antigen are not as sensitive as those obtained with the cholesterinized antigen these experiments offer little hope that the formaldehyd-gel test as understood at present will replace the Wassermann test for syphilis.

TABle 2.-Comparison of the Results Obtained by thf Alcoholic Extract Antigen and Formaldehyd-Gel Test with the Results Obtained with the Cholesterinized Antigen

\begin{tabular}{|c|c|c|c|c|c|c|}
\hline \multirow{4}{*}{ 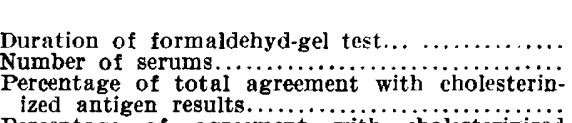 } & \multicolumn{3}{|c|}{ Aleoholic Extract } & \multicolumn{3}{|c|}{ Formaldehyd-Gel } \\
\hline & $-\overline{10}$ & $=$ & $-\bar{c}$ & 24 & 48 & 72 \\
\hline & 319 & 477 & 284 & 319 & 477 & 284 \\
\hline & 89 & 92 & 90 & 86 & 87 & 85 \\
\hline Percentage of agreement with cholesterinized & & & & & & \\
\hline $\begin{array}{l}\text { antigen, Wassermann negative..................... } \\
\text { Percentage of agreement with cholesterinized }\end{array}$ & 100 & 100 & 100 & 98 & 98 & 91 \\
\hline 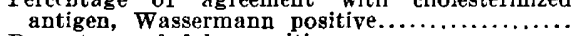 & 21 & 25 & 22 & 12 & 16 & 21 \\
\hline Percentage of false positives................... & - & 二 & 一 & 1.8 & 2.1 & 4 \\
\hline
\end{tabular}

There are two objections to the use of the formaldehyd-gel test as it is at present understood: First, it fails to give a positive reaction on the serums of many patients suffering with syphilis (our faise negatives). Second, it gives a small percentage of positive reactions on the serums of patients whose classification as syphilitics is doubtfui (some of our false positives). Until we have more data concerning these supposedly false positives and the specificity of the reaction, and can decrease the number of false negatives, we cannot draw any definite conclusions regarding the value of the test and interpretation of the results.

The formaldehyd-gel test gave positive reactions with 21 per cent. of the serums giving positive Wassermann reactions with the cholesterinized antigen. Therefore, it gave about 80 per cent. of false negatives. This percentage of false negatives would have been larger if we had adhered to the method used by Gaté and Papacostas. By eliminating the stoppers and incubating and reading the results at a later period, we were able to decrease the percentage of false negatives. A large majority of the patients represented by the 80 per cent. of false negatives were clinically syphilitic.

An examination of the clinical histories of patients giving false positives with the formaldehyd-gel test shows that some were clinically syphilitic, some of them had previously had a positive Wassermann 
test and been treated for syphilis and some gave a positive Wassermann reaction with icebox incubation. In some cases there were no clinical evidences of syphilis and other diagnoses had been made. In the remaining cases there was no means of determining whether or not the patient was syphilitic.

With the formaldehyd test at ninety-six hours and later, the increase in false positive reactions is much more rapid than the increase in true positive reactions. We have chosen the results obtained with the formaldehyd at seventy-two hours instead of thirty hours for our detailed comparison with the Wassermann test because of the increased number of true positives. There is an increase in the number of false positives, but at this period this is not out of proportion to the increase in true positives.

It is probable that desiccation plays a more important part in the coagulation of the serum at periods exceeding seventy-two hours. We have already described experiments which indicate that desiccation is a factor in the coagulation of the serums at periods under seventy-two hours. There is considerable evaporation in the incubator in ninety-six hours, and accompanying this evaporation there is a greatly increased percentage of false positives with the formaldehyd test. The prevention of desiccation by the use of stoppers may eliminate some of the false positives, but it also delays and reduces the percentage of true positive reactions. It is possible that some method of shortening the test or eliminating some of the false positives may be discovered by a more thorough study of the effect of desiccation. Associated with evaporation of the water there is, of course, volatilization of the formaldehyd gas.

In comparing our results with those obtained by Gate and Papacostas, it should be borne in mind that the tests were made on different serums, the Wassermann tests were made by a different technician; a different sample of commercial formaldehyd was used, and the tubes were incubated without stoppers, resulting in greater evaporation of water and volatilization of the formaldehyd gas. Gaté and Papacostas state that incubation does not affect the result. In our experiments the incubation without stoppers increased the number of formaldehydgel reactions.

Gaté and Papacostas obtained a total agreement of 85 per cent. We obtained a total agreement of 85 per cent. or higher, depending on the antigen used. The percentage of total agreement varies with the proportionate number of positive and negative serums used. If we had made the test on an equal number of positive and negative serums, the total agreement would probably have been reduced to about 60 per cent. because the formaldehyd-gel reactions are in closer agreement with the negative Wassermann than with the positive Wassermann reactions. 
When the reactions of the two tests differed, as they did in 15 per cent. of the cases, Gaté and Papacostas were unable to determine which of the two tests was the more accurate. In our experiments the clinical histories indicate that the Wassermann test, using a cholesterinized antigen and water bath incubation, is the more accurate. However, in some cases in which the results differed, the clinical - histories suggested that the formaldehyd-gel reaction gave the correct diagnosis. Gaté and Papacostas found this to be true of early syphilis. We were unable to verify this point.

INTERPRETATION OF FORMALDEHYD-GEL REACTIONS

1. When parallel Wassermann, cholesterinized antigen and water bath incubation, and formaldehyd-gel tests are made on serums:

If the Wassermann reaction is positive and the formaldehyd-gel reaction is negative, the patient is probably syphilitic.

If the Wassermann reaction is negative and the formaldehyd-gel reaction positive, the patient should be considered as possibly syphilitic, as in an undetermined percentage of cases the formaldehyd-gel reaction is correct. The Wassermann test should be repeated.

If both tests are positive the formaldehyd-gel reaction confirms the Wassermann diagnosis but does not prove that the patient has syphilis.

2. When only the formaldehyd-gel test is made:

The use of the formaldehyd-gel test to the exclusion of the Wassermann test for syphilis is not advisable. When it is impossible to make a Wassermann test, the physician should make a formaldehyd-gel test. Under such conditions the physician will not be tempted to make the test unless the patient has been exposed and gives clinical evidence of syphilis. Consequently, if the formaldehyd-gel reaction is positive, the chances are five or more to one that a Wassermann test would also be positive and that the patient is syphilitic. If the formaldehyd-gel reaction is negative, the chances are about equal or less than equal that the Wassermann test will give the same result. A positive formaldehyd test, under the conditions of general practice in which only suspicious cases are tested for syphilis, is of much greater diagnostic value than a negative formaldehyd test.

\section{SPINAL FLUID}

Formaldehyd-gel tests were made on seventy spinal fluids, eighteen of which gave positive Wassermann reactions. None of these spinal fluids gave positive formaldehyd-gel reactions. Various amounts of formaldehyd were added to the spinal fluids, and they were incubated for several days. 


\section{FUTURE RESEARCH}

A great deal more experimental work must be done before we can correctly estimate the value of the formaldehyd-gel reaction in comparison with the Wassermann test for syphilis, or suggest a satisfactory basis for the interpretation of results. Future research is needed with these objects in view:

1. The acquisition of more data on which to estimate the value of the test, as described by Gate and Papacostas or modified by us, and to form the basis for a more accurate interpretation of results and the specificity of the reaction.

2. The discovery of other methods of using formaldehyd gas in an effort to shorten the time required for the test, to eliminate false positive and negative reactions, and to determine whether all solutions of formaldehyd give the same result. The introduction of formaldehyd gas into the serum may give results more reliable than the use of a solution of formaldehyd. This is suggested by the fact that evaporation is a factor in the formaldehyd-gel reaction.

3. The determination of the effect of heat, evaporation, inactivation and volatilization of the gas. Our experiments were all made on inactivated serum. Gate and Papacostas state that inactivation does not affect the result. The process of inactivation increases evaporation, and we have shown that evaporation affects the result of the test. It remains to be determined whether the test can be shortened by preliminary boiling of the serums after the addition of the formaldehyd.

4. The discovery of other means, either chemical or physical, than the use of formaldehyd to cause a gel formation when added to positive serum. Serums vary in their coagulation time when exposed to heat. Possibly positive serums will coagulate at lower temperatures than negative serums.

5. The determination of the substance on which the gel reaction depends. The discovery of the nature of this substance may suggest other means than the gel reaction of determining the presence or amount of this substance in positive serums.

\section{CONCLUSIONS AND SUMMARY}

The formaldehyd-gel test as used in these experiments is not so reliable a test for syphilis as the Wassermann test with a cholesterinized antigen and water bath incubation. It is not quite so reliable as the Wassermann test with an alcoholic extract antigen.

The formaldehyd-gel test gives results in closer agreement with the negative than with the positive Wassermann tests. 
When the two tests disagree, a positive formaldehyd-gel diagnosis will be found to be correct in an undetermined percentage of cases; a negative formaldehyd-gel diagnosis will rarely be correct.

The substance on which the formaldehyd-gel reaction depends is not present in the spinal fluid of syphilitic patients, or at least not in sufficient amounts to cause coagulation on the addition of formaldehyd.

Incubation of the serums in unstoppered tubes increases the number of positive formaldehyd-gel reactions. The increase in the number of positive reactions accompanies and is probably dependent on increased desiccation. It will probably be found that inactivation, unless increased evaporation is prevented, also affects the percentage of positive formaldehyd-gel reactions.

Heat, aside from the effect on desiccation, does not increase the number of positive reactions. Serum to which a small amount of formaldehyd has been added does not coagulate on exposure to a boiling temperature in a test tube. The icebox temperature delays the coagulation of the positive serums.

An increase in the amount of formaldehyd added to the serums increases the percentage of water and delays the coagulation of the positive serums.

Suggestions are given for the interpretation of results obtained with the formaldehyd-gel test and for future research. 